\title{
Fluid-structure interaction study of stenotic flow in subject specific carotid bifurcation: a case study
}

\begin{abstract}
Advances in numerical simulation have allowed the investigation of complex interaction of blood flow through elastic arteries which can be useful in demonstrating the disease progression and haemodynamics of cardiovascular diseases such as atherosclerosis. In the present study, patient is diagnosed with an occluded right Internal Carotid Artery (ICA) with partial stenosis at root of right External Carotid Artery (ECA) and partial stenosis at the root of left ICA. 3D patient specific carotid bifurcation is generated based on CT scan data using MIMICS-14.0 and numerical analysis is performed using FSI in ANSYS-14.5. The blood flow is assumed to be incompressible, homogenous and Newtonian, while artery wall is assumed to behave linearly elastic. The two-way sequentially coupled transient FSI analysis is performed using FSI solver for three pulse cycles and haemodynamic parameters such as flow pattern, Wall Shear Stress (WSS), pressure contours and arterial wall deformation are studied at the bifurcation and critical zones. The variation in flow behavior is investigated throughout the pulse and simulation results obtained reveals that there is a considerable increase in the flow behavior in partially stenosed carotid unlike occluded carotid. The investigation also demonstrates disturbed flow pattern especially at the bifurcation and stenosed zone elevating the haemodynamic variation during peak systole and later part of pulse cycle. The obtained results agree well with the clinical observation and demonstrate the potential of patient specific numerical studies in prognosis of disease progression and plaque rupture.
\end{abstract}

Keyword: Arterial stenosis; Carotid artery bifurcation; Fluid-structure interaction; Wall shear stress 